ISSN: $1130-3743$

\title{
LA EFICACIA EN LA EDUCACIÓN A DISTANCIA: ¿UN PROBLEMA RESUELTO?
}

\section{The efficiency in distance education. A resolved problem?}

\author{
L'efficacité dans l'éducation à distance. Un problème \\ résolu?
}

Lorenzo García Aretio y Marta Ruiz Corbella

UNED. Facultad de Educación. Departamento de Teoría de la Educación y

Pedagogía Social. Paseo Senda del Rey, 7. 28040 Madrid. Correo-e: lgaretio@edu. uned.es,mruiz@edu.uned.es

Fecha de recepción: enero de 2010

Fecha de aceptación definitiva: abril de 2010

Biblid [(1130-3743) 22, 1-2010, 141-162]

RESUMEN

Gracias a la preocupación existente por la calidad de la educación a distancia, se ha reunido una amplia literatura científica que ha ayudado a detectar los puntos fuertes y débiles de esta metodología, lo que ha conducido a su permanente mejora. Una de sus líneas de investigación se dirige a comprobar si existen diferencias significativas entre la enseñanza presencial y la a distancia.

A través de la metodología empírica de análisis de contenido, hemos accedido a investigaciones sobre el rendimiento de los estudiantes en ambos formatos, con el objetivo de detectar diferencias significativas entre éstos. Este metaanálisis evidencia que la eficacia en la educación no depende de los recursos tecnológicos, ni de los propios profesores, ni de los estudiantes, aunque todos ellos son decisivos, sino de la calidad de sus diseños pedagógicos. Con diseños rigurosos, 
sea en formatos presenciales o en modalidad a distancia, los resultados no difieren significativamente.

Palabras clave: educación a distancia, educación presencial, eficacia, rendimiento, calidad de la educación, aislamiento.

\section{SUMMARY}

Thanks to the concern for the quality of distance education, a large amount of scientific literature had been produced to help detect the strong and weak points of this type of methodology and this has contributed to its constant improvement. One line of research focuses on whether significant differences exist between face to face and distance learning.

Using an empirical methodology of content analysis, we have explored research on student performance in both formats in order to detect major differences between the two. This meta-analysis shows that the effectiveness of the education does not depend on the technological resources available or the teachers or the students, although they are all decisive, but rather on the quality of pedagogical design. With rigorous designs, whether they be used in face to face scenarios or in distance education, the results are not significantly different.

Key words: distance education, face to face education, effectiveness, achievement, quality of education, isolation.

\section{SOMMAIRE}

Grâce à l'inquiétude suscitée par l'éducation à distance, concernant sa qualité, a été réunie une ample littérature scientifique qui a aidé à détecter les points forts et faibles de cette méthodologie, ce qui a mené à sa constante amélioration. L'une des voies de recherche, a pour objectif de vérifier s'il existe des différences manifestes entre l'enseignement présentiel et l'enseignement à distance.

À travers la méthodologie empirique de l'analyse de contenu, nous avons eu accès à des recherches sur le rendement des étudiants dans les deux types de formation, dans le but de détecter des différences significatives entre ces derniers. Cette métaanalyse met en évidence que l'efficacité dans l'enseignement ne dépend ni des ressources technologiques, ni des professeurs, ni des étudiants, quoique tous s'avèrent être décisifs, mais de la qualité de ses conceptions pédagogiques. Avec des conceptions rigoureuses, soit en format présentiel, soit dans la modalité à distance, les résultats ne diffèrent pas de façon significative.

Mots clés: éducation à distance, éducation présentielle, efficacité, rendement, qualité de l'éducation, isolement. 
1. ¿SE Sigue dudando de la eficacia de la Educación a distancia?

En 2009 acabamos de publicar un libro que muy conscientemente titulábamos ¿Por qué va ganando la educación a distancia? ${ }^{\text {. }}$. Con ese título damos por supuesto que, en efecto, estamos convencidos de que, en cualquiera de sus formulaciones, la modalidad, la metodología, las prácticas, las propuestas o proyectos de educación a distancia, han ido ganando terreno progresivamente y sin cesar, a lo que pudiéramos denominar como modalidad presencial o de docencia cara a cara sin mediación tecnológica.

Están arrebatando espacio y tiempo a las formas más convencionales de enseñar y aprender, las metodologías a distancia que priman el trabajo autónomo de los estudiantes, así como las actividades cooperativas y colaborativas donde estos mismos participantes aprenden con otros, de otros y para otros a través de las redes sociales, a través de comunidades de aprendizaje ancladas en soportes digitales o, lo que es más habitual, a través de plataformas virtuales diseñadas con fines docentes.

Así, quienes desde hace muchos años venimos abogando por el estudio, investigación, análisis, contraste y divulgación científica de las bases, fundamentos, estructura, métodos, posibilidades, etc., de la educación a distancia en sus diferentes presentaciones, observamos con satisfacción que, ciertamente, ésta va conquistando terreno. Cada vez hay un mayor número de instituciones, de programas, de docentes e investigadores comprometidos, de estudiantes que confían en esta modalidad y, lo que es más importante, los resultados sobre eficacia y eficiencia que se vienen mostrando aparecen al menos como de nivel similar a los de los procesos presenciales.

No fueron fáciles los primeros años en los que se implementaron las iniciales experiencias de universidades a distancia. Quizás nadie analizó con criterio, o pocos se refirieron con rigor a varias décadas de crecimiento de una enseñanza por correspondencia que posibilitaba el acceso a diferentes ofertas de formación cultural, profesional, reciclaje, etc. Esa enseñanza por correspondencia supuso el soporte de las primeras universidades a distancia que realmente tuvieron su origen en los movimientos de extensión universitaria nacidos en Estados Unidos en la década de los años sesenta del siglo XIX, y que diez años después se trasladaban a Europa. Prueba de ello es el sistema adoptado por la Universidad de Londres en 1836 (Kaye, 1981).

Pero, fundamentalmente, fue con el nacimiento de la Open University británica, al ofertarse sus primeros títulos universitarios a distancia, cuando surgieron las mayores voces de alarma contra estas propuestas innovadoras. A partir de esa fecha la expansión de esta modalidad ha sido inusitada. Sólo un año después de que la Open University (OUUK) iniciase sus enseñanzas se creó la Universidad

1. García Aretio (2009). 
Nacional de Educación a Distancia (UNED) de España. No tardarían en diferentes países en abrirse otras grandes universidades a distancia, que muy pronto superaron los 100.000 estudiantes. Muestra de estas megauniversidades, en término de Daniel (1996) son:

- $\quad$ University of South Africa (UNISA), Sudáfrica, creada en 1837.

- Centre National d'Enseignement à Distance (CENED), Francia, 1939.

- Korea Air and Correspondence University (KNOU), República de Corea, 1972.

- $\quad$ Sukhothai Thammathirat Open University (STOU), Thailandia, 1978.

- Anadolu University (AU), Turquía, 1982.

- Universitas Terbuka (UT), Indonesia, 1984.

- Indira Gandhi National Open University (IGNOU), India, 1985.

- $\quad$ Payame Noor University (PNU), Irán, 1987.

A pesar de esta expansión, muchos pusieron en entredicho la credibilidad de esta modalidad, de ahí que cada avance, cada propuesta debía justificarse siempre muy fundamentadamente. Así, constantemente, la educación a distancia tenía que someterse a la verificación de su calidad (Brown y Wack, 1999). Por ello, estas universidades desde sus inicios han sabido desarrollar programas para la evaluación de la calidad, estudios dirigidos a la autoevaluación, que les permitiesen conocer para qué sirven, a quién sirven, cómo sirven, cuáles son los frutos de ese servicio en contraste con los objetivos de su creación, etc., convencidos de que es éste el único planteamiento serio que abrirá el camino de futuras mejoras y de una mayor eficacia. A la vez que interesaba mostrar a la sociedad qué tipo de Universidad son, qué aspectos eran similares e idénticos a los de otras universidades presenciales, cuáles eran sus claves diferenciadoras y, de forma especial, evidenciar la calidad de sus diseños formativos (García Aretio, 1997).

Lógicamente, esta línea de investigación que ahora se aborda en todo centro educativo, para las instituciones universitarias a distancia, ha sido un tema permanente de estudio, lo que ha permitido contar con literatura científica suficiente y reconocida en torno a su calidad y resultados. Por eso, la aludida afirmación de "ganancia" no la sustentamos sólo en apreciaciones basadas en los años de experiencia en este campo, sino en la aproximación a numerosos trabajos que desde hace años confirman nuestro convencimiento.

Somos conscientes de que nos movemos en un campo, el de la educación a distancia, que muestra un núcleo y unos perfiles más complicados que la educación convencional plasmada en la relación física cara a cara. Sabemos de la dificultad de determinados análisis y prácticas incluso en entornos educativos presenciales y reconocemos también la existencia de ofertas educativas a distancia con baja o nula calidad. Sin duda, es inviable trasladar el diseño de una acción formativa presencial tal cual a un entorno a distancia, como se ha llevado a cabo en muchas ocasiones. Esto sólo nos habla del desconocimiento de la educación a distancia 
por parte de esos promotores y explica los fracasos producidos en muchas de estas propuestas. Igualmente, sabemos que planificar y desarrollar acciones educativas a distancia no resulta sencillo, y ello porque nos enfrentamos a un ámbito que podríamos definir como:

a) Emergente (aunque cada vez menos), a la vez que consolidado,

b) complementario a los esquemas presenciales,

c) complejo por los numerosos agentes y componentes que han de intervenir en su diseño y desarrollo,

d) problemático al exigir equilibrar y armonizar las numerosas corrientes y todos y cada uno de los múltiples elementos intervinientes,

e) heterogéneo en sus manifestaciones, por la diversidad de propuestas, formulaciones y tipos de educación a distancia que hoy aparecen como tales o con otros nombres que nosotros integramos en el genérico de educación a distancia,

f) con bases múltiples por la cantidad de campos del saber que inciden en esta modalidad, tales como la pedagogía, la psicología, la tecnología, la informática, la sociología, la economía, la política, etc., y

g) en permanente y rápida evolución que complica todo lo anterior al exigir constante adaptación a los nuevos avances, sobre todo tecnológicos, tanto desde la perspectiva institucional, como desde la organizativa y pedagógica de docentes y estudiantes.

Tras esta contextualización, el objetivo de este trabajo es doble, quizás triple. Por un lado, mostrar la eficacia de la educación a distancia. Y, por otro, aproximarnos a uno de los grandes obstáculos o desventajas que numerosas investigaciones han apreciado en esta modalidad, la de la separación profesorestudiante/s que se traduce o se percibe en muchas ocasiones como el sentimiento de soledad ante el proceso de aprendizaje. Finalmente, como tercera intención, trataremos de acotar este rasgo negativo, en sus justos términos, para incluso neutralizarlo, sobre todo al amparo de las posibilidades de las más avanzadas tecnologías de la información y la comunicación que culminan hoy con el software social de la Web 2.0.

Aunque para la elaboración de este artículo las fuentes consultadas han sido abundantes, no nos ha resultado especialmente problemático localizar las que refuerzan los postulados que defendemos desde hace dos décadas y media. Incluso hemos consultado más fuentes de las referenciadas en el trabajo, que han sido rechazadas por nuestra intención de seleccionar sólo algunas de las más relevantes.

Como puede apreciarse, un alto porcentaje de las fuentes citadas fueron consultadas en texto completo a través de Internet. En muchos casos porque los documentos se ofrecen directamente online, pero otras muchas fuentes fueron igualmente localizadas en Internet, pero mediante los servicios de suscripción con 
que cuenta la biblioteca de nuestra Universidad a través de $E B S C O^{2}$. Téngase en cuenta que estos servicios disponen en abundancia, además de revistas científicas y electrónicas de suscripción, con trabajos inicialmente publicados en formato impreso, que hoy han sido digitalizados. Ha de destacarse también que la Universidad a la que pertenecen los autores del trabajo cuenta con una buena biblioteca de libros y revistas relacionados con esta temática ${ }^{3}$. Acudimos, igualmente, a los servicios suscritos por nuestra Universidad, de préstamo interbibliotecario, en tres ocasiones. A todo ello debemos sumar la experiencia de uno de los autores de este artículo que viene trabajando sobre esta temática desde 1985.

\section{2. ¿ESTÁ PROBADO QUE LA EDUCACIÓN A DISTANCIA ES EFICAZ?}

Hace pocas décadas eran escasas las experiencias de educación a distancia sistematizada, sometidas a contraste con las realizadas en ambientes presenciales, con siglos a sus espaldas. Eran escasos los estudios serios que comparasen unas y otras prácticas. Pero hoy día, muchas de las universidades del mundo cuentan con programas a distancia soportados en tecnologías más o menos sofisticadas. Ello ha propiciado la realización de trabajos de investigación dignos de ser considerados. Así en la actualidad, nos encontramos con numerosa literatura científica que se genera en torno a las innovaciones que apuestan por formas diferentes de enseñar y aprender realizadas en contextos que antes eran exclusivamente presenciales. A ello se suma el aumento considerable de instituciones unimodales ${ }^{4}$, que imparten su oferta educativa con la metodología a distancia de forma exclusiva, así como de las duales o mixtas que progresivamente van incorporando cada vez más programas a distancia. Son de todos conocidos los campus virtuales existentes en tantas universidades presenciales, así como los consorcios de universidades que operan en este sentido.

Para evitar equívocos señalaremos que bajo el concepto de educación a distancia desde siempre venimos englobando a todas aquellas prácticas que hoy se vienen conociendo como enseñanza-aprendizaje abiertos, teleformación, enseñanza-aprendizaje virtual, online, e-learning, etc. Y ello, porque consideramos que todas esas formulaciones responden a las definiciones que desde hace dos décadas manejamos (García Aretio, 1986, 2001). Para poder reconocer si realmente estamos ante una metodología a distancia, ésta debe basarse en un diálogo didáctico mediado entre el profesor (institución) y el estudiante que, ubicado en espacio diferente al de aquél, aprende de forma independiente y/o cooperativa (García Aretio,

2. Se hace mención a esta circunstancia en las referencias bibliográficas.

3. Biblioteca del Instituto Universitario de Educación a Distancia (IUED) de la UNED.

4. Prueba de ello es que en España, a la UNED, www.uned.es y a la UOC, www.uoc.edu, se le han sumado recientemente, la UDIMA, www.udima.es, la UNIR, www.unir.net y la VIU, www.viu.es. 
2001). Así, todas esas formulaciones, denominaciones y modos de hacer, con todos los matices que se quiera, pueden considerarse como propuestas de educación a distancia, por hablar de un concepto superior al de mera enseñanza a distancia.

En este trabajo no pretendemos demostrar que la educación a distancia sea mejor que la presencial. El enfoque nunca debería ir por ahí. Pretendemos responder al epígrafe de más arriba. Es decir, si diésemos por supuesto que la educación presencial es eficaz, querríamos mostrar que la educación a distancia también lo es. Y para ello nos vamos a apoyar básicamente en uno de los indicadores habituales siempre que se abordan comparaciones de este tipo, el del rendimiento académico de los alumnos que siguen estudios en formatos presenciales o a distancia, el de sus calificaciones o puntuaciones, fruto de exámenes o pruebas.

Por eso, en respuesta al primer objetivo de este trabajo, queremos probar que determinados rendimientos académicos de estudiantes de una modalidad u otra no presentan diferencias significativas estadísticamente hablando (Clark, 1994). En realidad han sido múltiples los trabajos que concluyen que el rendimiento de los estudiantes depende más de los diseños pedagógicos de cada acción formativa que de los recursos seleccionados para el aprendizaje. Con diseños rigurosos, sea en formatos presenciales o en modalidad a distancia, los resultados no difieren significativamente. En definitiva, estamos con Clark, en que parece probado que el aprendizaje no es fruto de la tecnología, sino más bien del método pedagógico empleado. Aunque, la tecnología pueda abundar en la mejora de estos procesos, una vez que la integremos debidamente en el diseño pedagógico asumido.

Con anterioridad al estudio citado de 1994 el mismo autor, Clark (1983), apuntaba que con la introducción de cualquier tecnología en determinado proceso de aprendizaje se suele captar la atención del estudiante por la novedad del artefacto y artilugio utilizados, pero progresivamente los estudiantes se van acostumbrando al medio y ya una vez perdidos los iniciales valores motivacionales vuelve a contar más el diseño, el sentido y los principios pedagógicos.

Para confirmar nuestra postura, hemos consultado estudios, fundamentalmente revisiones y metaanálisis que han tratado de realizar algún tipo de contraste entre ambas modalidades. Quizás la mayor sistematización que se ha llevado a cabo respecto a estos estudios comparativos entre enseñanza a distancia y los métodos presenciales la desarrolló Russell, cuyo trabajo en su 5. a edición fue publicado en 2001. En este libro se recogen 355 estudios, informes técnicos, resúmenes, tesis y documentos de investigación desde 1928 hasta 1998 que utilizan metodologías cuantitativas, pero también cualitativas, así como análisis reflexivos o teóricos. Russell concluye que los resultados obtenidos por los estudiantes a distancia no son ni peores ni mejores que los obtenidos por sus colegas que siguieron procesos ordinarios de enseñanza-aprendizaje. En consecuencia, parece ser que la modalidad educativa no tiene efectos estadísticos significativos respecto a los resultados obtenidos por los estudiantes. 
Como una pieza complementaria al citado libro de Russell se ofrece el sitio Web No significant difference ${ }^{5}$. En esta Web, además de estudios e informes generados desde 1928, se facilita el acceso a los publicados o descubiertos en fecha posterior a la de la publicación del libro. En ese sentido, hemos revisado los estudios desde 1999 hasta los primeros meses de 2009 y los resultados son los siguientes: se recogen 68 estudios de los que 40 de ellos no muestran diferencias significativas, otros 24 dan resultados mejores para la enseñanza mediada por tecnologías y sólo los 4 restantes producen resultados favorables a la educación en el aula. Russell afirma que no pudiéndose constatar diferencias significativas evidentes, sí que parece que la tendencia es favorable a los estudios no convencionales. Sin embargo, ha de reseñarse que también concluye que no hay nada inherente en la tecnología que produce mejoras en el aprendizaje.

Es de destacar que el arco temporal de todas estas investigaciones ofrece una realidad permanente en el tiempo que, de forma invariable, manifiesta la similar eficacia de ambas modalidades, no dependiendo su eficiencia de recursos más o menos logrados, sino de su propio diseño pedagógico. Por otro lado, el número de investigaciones expone que los mejores resultados en una u otra modalidad dependen más de situaciones y experiencias concretas, en las que sobresale la novedad de una tecnología o la mejor adecuación a una materia concreta de una metodología específica, o la participación decisiva del profesorado, o... Pero estas situaciones excepcionales no avalan la preponderancia de uno u otro diseño. Sin embargo, en los trabajos en los que se constatan diferencias significativas evidentes, sí se da una tendencia favorable a los estudios basados en la incorporación de recursos tecnológicos en la enseñanza.

En consecuencia, y como ya señalábamos más arriba, el mayor número de investigaciones proporcionadas por Russell evidencian que la eficacia en la educación no depende de los recursos tecnológicos, ni de los propios profesores, ni de los estudiantes, aunque todos ellos son decisivos, sino de la calidad de sus diseños pedagógicos. De ahí que no exista una diferencia significativa entre ambas modalidades. Sin embargo, no nos damos por satisfechos con esta minuciosa revisión de Russell.

Para ahondar en nuestro propósito, hemos manejado la herramienta suscrita por nuestra biblioteca EBSCO $A$ to $Z^{6}$. Como complemento ideal, hemos utilizado el potente sistema de referencias, EBSCO-Host ${ }^{7}$. Tras los años de trabajo en todo

5. La dirección de esta página es www.nosignificantdifference.org.

6. EBSCO A to $Z$ permite la localización de una ingente cantidad de recursos electrónicos, incluidos publicaciones electrónicas, títulos en bases de datos de textos completos, paquetes editoriales y libros electrónicos.

7. EBSCO-Host consiste en un sistema de referencias bibliográficas a través de Internet, que ofrece una variedad de especialidades de bases de datos de texto completo y bases de datos populares de 
lo relacionado con la educación a distancia, tenemos configurado My EBSCOhost . $^{8}$ Con motivo de este trabajo, hemos intensificado algunos perfiles de búsqueda. Las búsquedas las hemos centrado en ERIC (la fundamental) y también en MLA International Bibliography, PSycARTICLES, SOCINDEX y E-Journals. Las posibilidades de búsqueda son inmensas. Además de las carpetas ya almacenadas desde hace tiempo en My EBSCOhost, hemos insistido, siempre referido a la educación a distancia en sus diferentes formulaciones, en términos tales como meta-analysis, research, difference, significant, review y effectiveness.

De los resúmenes de resultados que posteriormente referimos, algunos de ellos han sido recogidos también por Russell. De su libro citado hemos analizado unos pocos, los que entendimos como más relevantes para nuestro propósito, los que a lo largo de los años hemos valorado como más citados por otros autores que se acercan a esta temática y, además según nuestro entender, que quedaban avalados por una investigación rigurosa. Pero también en esos casos, siempre revisamos fuentes primarias que no nos fue complicado conseguir como ya señalamos anteriormente. Una vez seleccionada la fuente y contrastada su fiabilidad y validez, nos hemos limitado a ofrecer un mínimo resumen de sus resultados.

Dentro de los estudios presentados por Russell, se seleccionaron aquellos que, a su vez, eran revisiones de trabajos relacionados con el tema. Al margen de éstos, se seleccionaron otras nueve revisiones. En fin, entre todos esos trabajos relacionados con nuestro primer objetivo, hacemos mención sólo a estos pocos que nos parecen relevantes por las razones ya señaladas y suficientes para nuestro propósito en esta primera parte de este artículo.

En 1962 Schramm, de la Universidad de Stanford, llevó a cabo una investigación sobre la eficacia del aprendizaje a través de la televisión en la que revisó 393 trabajos. En este metaanálisis concluyó que en 255 de ellos no se evidenciaba una diferencia significativa entre la propuesta formativa a través de la televisión y la llevada a cabo de forma presencial.

En 1990 Bajtelsmit aporta, a partir de la revisión de 5 estudios, que son comparables, los resultados académicos logrados en ambos modelos de enseñanza, aunque si revisamos el criterio de coste y de economía de escala, sin duda, la educación a distancia sale beneficiada. Más tarde, en 1995, Barry y Runyan revisan las experiencias formativas llevadas a cabo en instituciones militares de Estados Unidos. En todas ellas no se ve una diferencia significativa entre los estudiantes a distancia y los que cursaron sus estudios de forma presencial.

los principales proveedores de información. EBSCO-Host permite realizar búsquedas simultáneamente en diferentes bases de datos.

8. My EBSCO-Host es un servicio personalizado que permite guardar preferencias, organizar la búsqueda en carpetas, guardar y recuperar el historial de búsquedas y obtener acceso a investigaciones guardadas de forma remota. 
Si nos fijamos en el trabajo de Moore y Thompson (1997), se llegó a la conclusión, después de la revisión de la literatura producida entre los años 1980 y 1990, de que la educación a distancia era eficaz cuando esta eficacia se medía por el rendimiento académico de los estudiantes, por las actitudes de éstos y sus profesores, y, de forma especial, por su economía de escala.

En el 2000 Saba insiste en que desde la década de los 50 se están llevando a cabo estudios comparativos entre la enseñanza a distancia y la presencial, y la realidad, de forma tozuda, viene evidenciando la no diferencia significativa entre ambas formas de instrucción.

Dos años más tarde, Hiltz, Zhang y Turoff (2002) examinaron diecinueve investigaciones que comparaban los estudios presenciales y los de educación a distancia, de carácter asíncrono y encontraron que cuando los resultados eran fruto de las consultas realizadas a los estudiantes, en general no se dedujeron diferencias significativas, sin embargo, cuando los resultados fueron fruto de sistemas de recopilación de datos objetivos, tales como exámenes, calificaciones, etc., encontraron mejores resultados en los estudiantes a distancia. Otro estudio que destacamos es el de Rivard (2002), que se basa en la comparación de los logros académicos de estudiantes presenciales y a distancia a lo largo de cuatro años. Los resultados fueron significativamente más altos para los que cursaron estudios a distancia.

Otro estudio a destacar es el de Shachar y Neumann (2003), que realizaron un metaanálisis a partir de 86 estudios distribuidos entre 1990 y 2002. La conclusión que se extrae favorece la hipótesis de un rendimiento académico superior por parte de los estudiantes que habían seguido cursos a distancia.

También debemos destacar una revisión de estudios sobre esta temática en la que se abordaban cuestiones relacionadas además de con los logros académicos, con las actitudes y las tasas de retención. Se basó la revisión citada en 232 trabajos empíricos referidos a diferentes niveles del sistema educativo y realizados entre 1985 y 2002 (Bernard y otros, 2004). Aquí se vuelve a reiterar la importancia de los diseños pedagógicos aplicados en los cursos, por encima de los recursos tecnológicos utilizados, y de si se enseña a distancia o cara a cara. Parece que en cuanto a los resultados las diferencias entre los procesos presenciales y a distancia, cuando estos últimos son síncronos, favorecen levemente a los estudios presenciales tradicionales, sin embargo, esa leve diferencia significativa favorece a los estudios a distancia cuando éstos se realizan en formato asíncrono. Y debemos recordar que toda educación a distancia tiene su máximo componente en relaciones asíncronas.

Finalmente, reseñamos una reciente publicación del Departamento de Educación de Estados Unidos (Means, Toyama, Murphy, Bakia y Jones, 2009), en la que se realiza una búsqueda sistemática de la literatura de investigación desde 1996 hasta julio de 2008. En esa búsqueda se identificaron más de un millar de estudios empíricos de aprendizaje en línea. La selección de estos estudios se basó en el contraste con la enseñanza presencial, en la medida de los resultados de aprendizaje. De todos éstos se seleccionaron 51 estudios para ser objeto de un metaanálisis. 
Éste arrojó el resultado de que, como promedio, los estudiantes en el aprendizaje en línea mostraron mejores resultados que los que recibieron programas cara a cara. Por otra parte, fueron superiores a ambas prácticas, los resultados de aquellos estudiantes que siguieron la modalidad de blended-learning (aprendizaje mezclado, mixto, semipresencial o combinado de docencia en línea y presencial). Aunque el objeto de la investigación era sobre estudiantes de enseñanza secundaria, la realidad fue que sobre este nivel existían pocos estudios y en los que se analizaron, los resultados no fueron tan favorables a estas nuevas modalidades. A partir de esta conclusión se infiere que la edad de los estudiantes es también una importante variable a considerar.

No vamos a negar que existan, en efecto, estudios en sentido contrario, que pueden mostrar mejores resultados de los estudiantes que siguen propuestas de corte convencional. Sin embargo, es cierto que abundan más los resultados en los que no se detectan diferencias significativas, aunque, como hemos señalado, muy centrados en los resultados y las calificaciones de determinadas pruebas? ${ }^{9}$. Todo esto nos lleva a afirmar que no debemos ser dogmáticos en la afirmación de que sólo pueden adquirirse aprendizajes de calidad mediante formatos tradicionales cara a cara, ni pasarnos al bando de los que piensan que actualmente todos los cursos sostenidos en sistemas digitales son claramente superiores con respecto a los resultados de aprendizajes valiosos por parte de los estudiantes.

Ya de los primeros trabajos recogidos por Russell en su recopilación y revisión de referencia, y de otros posteriores, puede concluirse que la incorporación a la docencia de diferentes recursos tecnológicos (radio, televisión, audio, vídeo, etc.) no dañaba a los aprendizajes de los estudiantes, es decir, no se producían diferencias significativas entre unos y otros, generalmente ni en un sentido ni en el otro.

Sin embargo, al igual que este autor, nos sentimos desilusionados al comprobar la creencia de una gran cantidad de docentes, directivos y técnicos que continúan pensando que la utilización de tecnologías para el aprendizaje es, por el uso en sí de esas tecnologías, causa directa de la calidad de estos aprendizajes. También existen quienes continúan afirmando lo contrario. Las creencias y las teorías implícitas siguen prevaleciendo, cuando las investigaciones nos muestran que no hay una modalidad de enseñanza superior a otra, al menos en los estudios superiores. Cuando lo que debemos tener en cuenta a la hora de escoger una u otra modalidad es definir claramente a quién va dirigida, qué objetivos se quieren lograr, con qué recursos se cuenta, etc. Por tanto, los rendimientos no dependen de la modalidad o de los recursos empleados en el desarrollo de una determinada acción formativa o curso. Es decir, los estudiantes a distancia no salen perjudicados en sus aprendizajes por seguir un curso en modalidad diferente a la convencional, que era en definitiva la crítica que se venía haciendo de la metodología a

9. Al menos, es lo que nosotros hemos localizado. Aunque sí hemos encontrado pocos, no conocemos estudios en una cantidad y calidad, similares, que muestren lo contrario. 
distancia al tratarla como una enseñanza de segundo nivel, una opción de calidad claramente inferior. Así, está de más el preguntar si pueden o no los estudiantes adquirir a distancia aprendizajes de calidad. Entendemos que más beneficioso sería plantearse:

- los efectos que determinados recursos tecnológicos pueden generar en los aprendizajes,

- qué tecnología para qué estrategia didáctica concreta,

- qué tecnología para qué contenidos,

- qué recursos o medios para qué metodología,

- cómo aprovechar mejor las tecnologías según los objetivos pretendidos, etc.

Bien es cierto que se han vertido críticas al conjunto de las investigaciones realizadas en este sentido, en unos casos por la debilidad de los propios diseños (Moore y Thompson, 1997) y en otros por la referencia al control de diferentes variables (Basu, 2005) que generalmente denominamos como "no intervinientes", pero que en realidad juegan un papel importante en cualquier investigación en el ámbito educativo, ¿cómo prescindir de las características del estudiante, de su entorno, de los métodos, de los materiales, del propio profesor, de los compañeros, etc.? (Phipps y Merisotis, 1999). Pero eso sucede en toda investigación de corte educativo (Brown y Wack, 1999). Por otra parte, en algunas de éstas se echan de menos las bases teóricas de carácter educativo en que se basan (Lockee, Moore y Burton, 2001). Una afirmación, según nuestra opinión, muy genérica y poco matizada dado que en las diferentes revisiones se ha cuidado en seleccionar aquellos trabajos mejor fundamentados y más rigurosos. Y, al menos, en las traídas a colación por nosotros, hemos extremado este cuidado.

\section{3. ¿SOLEDAD O INTERACCIÓN SOCIAL?}

Por lo expuesto hasta ahora podríamos preguntarnos, ¿es que no existen inconvenientes en esta modalidad a distancia? En buena parte de los estudios que abordan esta problemática se infiere que las tasas de abandono, fracaso o deserción son más altas en los formatos a distancia. Ello ha venido considerándose sobre todo referido a una educación a distancia de corte más convencional. Sin duda, el sentimiento de soledad del estudiante es una realidad, especialmente al inicio del estudio con esta metodología. Se trata de un alumno que físicamente se encuentra solo ante unos materiales y unos recursos y en un entorno muchas veces poco favorecedor para el estudio. A la vez, no todos sabemos ser, sin más, estudiantes a distancia. Las estrategias necesarias para el estudio a distancia difieren de las presenciales, y el grado de autonomía del estudiante es sensiblemente mayor desde el inicio de su proceso de aprendizaje, lo que conlleva unas destrezas específicas que deben adquirirse. Sin duda, la irrupción de las 
tecnologías colaborativas parece que vienen frenando esta falla de los estudios a distancia. Además, es una realidad que la creación de comunidades virtuales entre los estudiantes disminuye el abandono de éstos, aumentando, en consecuencia, su grado de satisfacción (Davies y Quick, 2001).

Así, abordando ya el segundo de los objetivos de este trabajo, señalaremos que numerosas investigaciones, la mayoría diríamos, de las que se han aproximado a averiguar las desventajas o problemas fundamentales de la educación a distancia, especialmente cuando se han realizado consultas a los estudiantes, destacan precisamente el sentimiento de soledad y de falta de comunicación a la hora de construir el conocimiento. Ello lo hemos podido constatar en investigaciones relacionadas con la UNED como después señalaremos (García Aretio, 1997). Este sentimiento de soledad, curiosamente, se deriva a partir de uno de los rasgos principales que definen a la educación a distancia: la separación física de profesores y estudiantes y de éstos entre sí.

En realidad nos encontramos con un dilema de difícil solución. El concepto de separación física es recogido por buena parte de las definiciones de educación a distancia que reconocidos teóricos de la misma vinieron realizando a lo largo de las últimas décadas del siglo pasado ${ }^{10}$. Es decir, un elemento medular de lo que entendemos que es la educación a distancia, la más convencional y la más moderna, es la consideración de que las conductas del docente y del estudiante, como tales, se desarrollan en espacios físicos diferentes o, si tuvieran lugar en el mismo escenario, de manera asíncrona. Así es que la paradoja surge cuando tantas investigaciones insisten en que el problema fundamental de la educación a distancia es el de la separación del estudiante de aquello que se supone es el contexto físico propio de los procesos de enseñanza-aprendizaje.

Esa separación no se vería como negativa si no fuese porque tras ella los estudiantes consultados la perciben con serios sentimientos de soledad. La mayoría de las investigaciones que se realizaron en la UNED durante sus primeros veinticinco años de existencia señalaban a esa "soledad" como una de las trabas más reiteradas por los estudiantes (García Aretio, 1997) ${ }^{11}$. Por otra parte esa separación, esa soledad, se traduce también en la base de otra de las características más destacadas de esta modalidad educativa, la independencia del estudiante y más que eso, su autonomía en el estudio. Quizás son dos rasgos que vienen forzados por la aludida separación pero que suponen principios educativos que bien manejados y equilibrados con los de carácter más interactivo son referentes propios de

10. Véanse al efecto los propios estudios de recopilación que al respecto realizamos (García Aretio, 1986, 1994, 2001).

11. En este libro se recoge un resumen de cada una de las más destacadas investigaciones realizadas hasta 1996 sobre la UNED de España, muchas de ellas fueron producto de sendas tesis doctorales. 
la educación de calidad dado que su puesta en práctica y producto final resultan altamente positivos.

Parece que a través de las conclusiones de las investigaciones referidas a la eficacia de la educación a distancia se avalarían parte de nuestros presupuestos iniciales, decimos "parte" porque al referirnos a la "ganancia" otra cuestión sería plantearse, además del rendimiento académico, la calidad de las interacciones humanas dentro de esta modalidad. Éste parece que puede ser el quid de la cuestión, el quid de la diferencia, ¿cómo lograr beneficios educativos relacionados con objetivos centrados en la formación social de los sujetos, similares a los que se obtienen en entornos cara a cara? En esto coincidimos con Tait (2000), en que las tecnologías colaborativas deben dar respuesta a esa cuestión. Es lógico que, al igual que otros muchos, este autor reconoce que la enseñanza en línea no puede suplir ese toque personal que supone la relación presencial entre profesor y estudiante, aunque sí que favorece la interacción, llegando en muchas ocasiones a situaciones de relación personal mucho más estrechas que las que suceden en formatos presenciales.

Pero veamos y acerquémonos a las últimas décadas del siglo pasado. La realidad de los estudiantes nuevos, los que accedían por primera vez a un sistema a distancia, sin experiencia anterior en este tipo de aprendizajes, es la de que arrancaban sus estudios con la ausencia de los compañeros que habitualmente les habían acompañado en todas las anteriores andaduras educativas. Esa "soledad", más allá del perjuicio que pudiera suponer por la ausencia de debate, de ideas diferentes, de opiniones encontradas relativas a los temas de estudio y, sobre todo, de interacción con sus iguales, parece que suponía un hándicap especial que les "invitaba" a abandonar los estudios (Paul, 1990). Y ello, además, por la falta de referente ante determinadas dificultades que ofrecen los materiales de estudio en esta modalidad. En un aula convencional resulta fácil e inmediato para un estudiante que no haya entendido las explicaciones del profesor, el contrastar, por ejemplo, que los demás tampoco las entendieron. Eso relaja y hace entender que el fallo no es personal. Por el contrario, el alumno a distancia más convencional ante la dificultad podía concluir que no entiende porque no llega, porque le faltan conocimientos previos, porque sus capacidades intelectuales no están al nivel exigido por estos estudios y esta frustración puede impulsar al abandono (García Aretio, 1987).

Existen alumnos que acceden a los estudios a distancia por el interés que tienen de aprovechar las ventajas de esta modalidad, la flexibilidad tempoespacial, la libertad para llevar el propio ritmo de aprendizaje, la autonomía e independencia que te brinda el acceso al conocimiento, etc. Sin embargo, hay otros que acceden a este tipo de formación porque no les queda más remedio, porque no existen otras vías, por dificultades económicas, familiares, de residencia, etc., para acceder a determinados estudios. En este último caso, se han encontrado estudiantes con mayores tendencias de desconcierto ante la falta de contacto y relación social con los compañeros (Krajnc, 1988).

Por tanto, insistimos, entre las características o rasgos de la educación a distancia más reiterados a través de las diferentes definiciones seleccionadas, la separación 
profesor-alumno es uno de los más destacados, junto con la ventaja de la posibilidad de favorecer el "estudio independiente". Pero este estudio independiente del alumno lo podemos considerar desde dos perspectivas, independencia como proceso o independencia como resultado. En el primer caso aludimos a lo que más podría criticarse y que podría entenderse como estudio "individual", aislado de los pares, lo que dificulta el logro de los objetivos relativos a la socialización. Ésta sería la parte negativa de este proceso. Ahora bien, esa "independencia" como "resultado" sería un objetivo de alto valor. Se trataría de lograr que el estudiante se preparase para ser independiente y autónomo, tanto en el aprender a aprender por sí mismo, como en la autonomía como persona que decide y es dueña de sí. Sin embargo, también debemos mencionar que esa soledad que se ha dado, y se sigue percibiendo, en los diseños de educación a distancia, está disminuyendo sensiblemente, como después veremos, gracias a la integración de las tecnologías de la comunicación y la información en estos mismos diseños. Por tanto, a la hora de criticar o de argumentar en un sentido o en otro, deben considerarse todos estos extremos.

En los sistemas a distancia de corte más convencional, digamos los ligados a la época de esplendor emergente de esta modalidad, décadas de los años 70 y 80 del pasado siglo, nunca se despreció el componente de interacción entre profesores y estudiantes y de éstos entre sí, ya fuese a través de los programados encuentros presenciales propios de estas universidades, como la Open University del Reino Unido o la UNED de España y que, por ejemplo, en el diseño de la Open University holandesa fueron una auténtica preocupación (Chang, 1983) con el fin de paliar esta limitación de la enseñanza a distancia; fuese a través de otros medios y sistemas de comunicación propios de la época: correo postal y teléfono, fundamentalmente, y en menor medida, fax, audio y vídeo. A pesar de ello, en 1996, Keegan alertó de la dificultad para lograr el equilibrio entre la pregonada independencia del estudiante en la educación a distancia y la necesidad de una interacción con los otros estudiantes. Buena parte del éxito de esta modalidad estaría en adecuar convenientemente dicha armonía, punto que, poco a poco, se ha logrado y a unos niveles insospechados.

Esta realidad se evidencia también a través del análisis de los recursos tecnológicos más utilizados en estas experiencias, que inicialmente fueron la televisión y el vídeo, y en menor medida, la radio. Ya a partir de los 90, las investigaciones dirigieron su mirada a los nuevos recursos que se incorporaron de forma masiva a la planificación de las acciones formativas a distancia. Estamos hablando ya del caso del correo electrónico primero y la Web después. Nadie pone en duda el enriquecimiento que desde esos momentos disfrutó la interrelación entre personas residentes en lugares distintos y distantes. Esas interacciones suponían en parte la superación de la soledad a que acabamos de hacer alusión. Los avances tecnológicos cuando han ido integrándose en estos procesos, una vez logradas sus posibilidades de transmisión, han avanzado hacia las facilidades para la comunicación, interacción y, finalmente, colaboración. 
Por otra parte, además de las ventajas que para algunos tiene un cierto anonimato a la hora de poderse expresar, otras investigaciones (Weiler, 1991) señalan que la distancia física proporciona, si se sabe utilizar adecuadamente, oportunidades para la reflexión y el análisis crítico, sin la cercanía, coactiva a veces, de un profesor. La reflexión, la contemplación de las ideas ofrece más oportunidades para asentar los aprendizajes que el "aquí y ahora" del aula. Son éstas las ventajas de las interacciones asíncronas que más arriba destacábamos como muy positivas.

Además, el anonimato de los alumnos, o al menos la no visión física de los mismos por parte de otros, en algunos procesos en línea hace que determinados estudiantes se sientan más cómodos, más libres, para poder expresar sus dudas, sus sentimientos, sus dificultades... También son muchos los estudiantes que manifiestan una mayor capacidad para expresarse en grupos de debate soportados en sistemas digitales (Chester y Gwynne, 1998; Chambers, 2002).

Otra crítica a la educación a distancia destaca que ésta no admite suficientemente la deliberación, el debate y contraste de pareceres entre profesores y estudiantes y de éstos entre sí. Matthews (1999) piensa que el valor lo proporciona el estar físicamente en un campus universitario e interaccionar social e intelectualmente con otros estudiantes y profesores. La ausencia de relación en el mismo espacio físico y de forma síncrona se ha observado por parte de los más críticos con preocupación y, en muchos casos, como motivo de alarma dado que se supone que esa falta de relación no prepara a los jóvenes para la participación ciudadana (Resnick, 2000). Pero este autor realiza estas críticas incluso para la denominada universidad virtual del siglo XXI que estaba llegando.

\section{CON EL SOFTWARE SOCIAL, MÁS INTERACCIÓN}

En todo caso, algunos de los críticos de los sistemas a distancia que basan sus argumentos en esta falta de interacción humana se detienen muy poco en la interacción real que existe en muchos formatos presenciales, más allá de los momentos informales de relación interpersonal entre estudiantes. De hecho resulta evidente que las posibilidades de interacción personal frecuente, uno a uno, entre profesor y estudiante se hace mucho más viable en sistemas en línea. Y afirmamos que también resulta así la relación entre todos los iguales, siempre que se disponga de herramientas de comunicación adecuadas.

Son bastantes las propuestas de educación a distancia que mantienen la preocupación de incorporar tecnologías con el fin de facilitar todas las posibilidades de interacción entre todos los actores y agentes de ese espacio formativo. De ahí que conforme han ido surgiendo tecnologías que han favorecido estas relaciones, la educación a distancia las haya ido incorporando. Y ésta es la dinámica que se está siguiendo en la actualidad con la Web 2.0.

En lo que nos afecta a nuestro interés han sido vitales los años que llevamos recorridos de este siglo XXI en los que las herramientas tecnológicas se han ido 
aproximando cada vez más a la facilitación de las relaciones sociales. El objetivo de este esfuerzo se ha centrado en lograr la interacción entre todas las personas, consiguiendo cada vez más y mejores niveles de comunicación, independientemente de los tiempos y espacios de cada uno (Levin, 2004). Si la educación a distancia ya se mostraba eficaz en las décadas finales del siglo pasado, a pesar del problema real de la escasez de interacción, qué podrá decirse en los años en que surge el software social, las redes sociales, la Web 2.0. Porque de lo que deberíamos hablar es de si puede o no existir en estos contextos la denominada presencia social, que entendemos como la capacidad de los alumnos a proyectarse social y afectivamente en una comunidad a través de las herramientas o software social (Rourke, Anderson, Garrison y Archer, 2001).

Como parece deducirse fácilmente de su denominación, el software social agrupa todas aquellas herramientas de comunicación basadas en Internet que facilitan la interacción entre personas y dan soporte a la configuración de redes sociales. Y en un contexto educacional, Anderson (2005) destaca que este software apoya a los estudiantes y los estimula a aprender juntos, manteniendo al mismo tiempo las características de independencia que posibilitan el control individual sobre su tiempo, espacio, presencia, actividad, identidad y grado de relación.

El software es social porque además de facilitar la comunicación, a través del mismo, los usuarios pueden contribuir con sus contenidos por lo que entre todos van construyendo el conocimiento y enriqueciendo sus aportes (Styles, 2006). Los trabajos en grupo, las comunidades, las comunicaciones en todos los sentidos, también denominadas herramientas o tecnologías colaborativas, facilitan tareas inimaginables en estos contextos educativos a distancia, gracias a las herramientas de mensajería de Internet, al chat, foros, blogs, e-portfolios, podcasting, wikis, redes sociales, peer-to-peer, etc. Son todas ellas componentes destacados de la Web 2.0 (Bragg, 2007).

Recordemos que la Web 1.0, la de siempre, presenta un carácter más transmisivo, ya que los estudiantes acceden al contenido creado por otra persona, pero no interaccionan con él. Sin embargo, en la Web 2.0 los estudiantes pueden diseñar sus propios contenidos (Arsun y Kuru, 2006). Parece que lo que realmente se quiere significar con esta Web no es otra cosa que la imparable evolución de Internet hacia cotas cada vez mayores de interacción y, sobre todo, de colaboración. Y además se incluye un cambio significativo en la interacción en esta Web, ya que hablamos de actitudes más que de herramientas o software (Davis, 2005), de hecho, más que tecnologías se definen comportamientos, aunque bien es cierto que para activar ciertas actitudes deberemos facilitar los entornos donde éstas puedan expresarse. Pero éstos, apoyados en un determinado software, han de entenderse como servicio más que como un producto.

Así, las contribuciones del usuario son las que en la Web 2.0 van construyendo la propia Web y, como consecuencia, el conocimiento. Desde nuestro punto de vista el ejemplo más paradigmático hoy es la Wikipedia de la que el propio creador del término Web 2.0, O’Really (2005), decía que era como una experiencia radical 
de confianza donde el saber se construye libremente por parte de los propios usuarios transformándose cada uno de simple usuario, de consumidor, en codesarrollador. ¿Cabe mayor democratización del conocimiento, no sólo en el acceso sino también en la creación? El contrapunto a esta experiencia de construcción colaborativa del conocimiento es la validez y fiabilidad del mismo.

Es la inteligencia colectiva (Lévy, 2004) la que supone el gran beneficio de esta nueva ola digital. En definitiva, hacen falta aplicaciones Web, software social, que diseñan y articulan otros con el fin de que los usuarios se configuren como protagonistas de la producción, creación, gestión, actualización y organización de contenidos a través de la interacción y colaboración grupal. Ante esta realidad, los educadores nos encontramos con posibilidades realmente atractivas para facilitar a los estudiantes el logro de los objetivos de aprendizaje. Estas posibilidades permiten una mayor independencia al estudiante, una mayor autonomía, pero a la vez mayores posibilidades de colaboración, de socialización, y de aumento de la eficiencia pedagógica (Franklin y Harmelen, 2007). También se define a la Web 2.0 como la Web de lectura y escritura (Richardson, 2006), y ello porque parece que va más allá de la prestación del servicio de lectura o mera visión y de descarga de contenidos, cuestiones propias de la Web 1.0. Ahora los lectores pueden también conformar y reescribir el contenido. En realidad existen muchos autores convencidos de que el fenómeno Web 2.0 es el aspecto de la sociabilidad que tiene el mayor potencial para mejorar la educación y, en concreto, una de las mayores posibilidades para la educación a distancia (Boyd, 2007). Y, sin duda, esta Web, al promover una mayor colaboración entre los estudiantes y entre profesores y estudiantes, mejora los logros de aprendizaje, la socialización y, consecuentemente, el grado de satisfacción (Barkley Cross y Major, 2005).

Sí es cierto que cuando surgieron teorías del aprendizaje como las conductistas, cognitivistas y constructivistas, las tecnologías de la información y la comunicación no se habían desarrollado como para impactar esos postulados teóricos. Pero, respecto a la integración de las tecnologías, todas ellas están ahora superadas por la teoría conectivista (Siemens, 2004) como teoría del aprendizaje para nuestro tiempo que trata de proporcionar entendimiento de las habilidades y tareas necesarias para que los aprendices prosperen en una era digital basada en redes. Pero insistimos, tanto en un caso como en el otro, todas estas teorías pedagógicas de antes se mantienen, aunque evolucionan, y lo que hacen es tratar de aprovechar las facilitaciones que las tecnologías ofrecen para la aplicación de éstas y de los principios pedagógicos que postulan.

\section{Conclusión}

Sin duda, los estudiantes en estos momentos, en todo entorno educativo a distancia, son realmente protagonistas de la propia construcción, gestión e, incluso, control de su saber y del acceso al mismo. Saber qué se necesita aprender en un momento determinado, dónde obtener los datos y la información precisa para ese 
aprendizaje, cómo procesar esa información para transformarla en conocimiento, cómo relacionarla, refundirla, recrearla, gestionarla, mostrarla, etc., en interacción permanente con los otros. En esta línea diferentes investigaciones concluyen que estas tecnologías están facilitando un aprendizaje más eficaz con un potencial de apoyo al desarrollo de competencias antes reservadas a los formatos presenciales (Klamma y otros, 2007). De ahí que podamos concluir que esa "soledad" del estudiante que hemos mencionado queda más que olvidada en los nuevos formatos digitales de enseñanza-aprendizaje.

A esa conclusión final, que responde al tercero de los objetivos que nos marcamos al inicio de este trabajo, debemos agregar el logro de los otros dos objetivos, el hecho de que creemos probada la eficacia de la educación a distancia, al menos a niveles similares a los de los formatos presenciales, así como que el inconveniente de la "soledad" del estudiante se ha ido viendo cada vez más reducido conforme las tecnologías han ido integrándose en estos procesos formativos.

Decididamente, nos reiteramos en las consideraciones iniciales de este artículo en el que partíamos del supuesto de que la educación a distancia "va ganando". Mientras estas formas diferentes e innovadoras de acometer estrategias para la docencia se sostengan en controles y garantías de calidad, soporten sus propuestas en diseños contrastados y partan de presupuestos teóricos y principios pedagógicos, no van a ser fácilmente frenadas por quienes aún en estos años dudan de la eficacia y eficiencia de esta modalidad o, más aún, reniegan de ella. Aunque bien es cierto que parece que hoy en día planteamientos serios son escasos en este último sentido, dado que parece probado que con diseños pedagógicos rigurosos los aprendizajes pueden ser igualmente de calidad, se produzcan éstos en formatos presenciales o a distancia.

Entendemos, por ello, que a estas alturas está fuera de lugar debatir si se pueden o no lograr resultados académicos de calidad mediante la educación a distancia. La respuesta es afirmativa si los objetivos de aprendizaje están claros y los diseños de metodología, recursos, evaluación, etc., son coherentes entre sí y se ajustan a parámetros pedagógicos de calidad.

\section{REFERENCIAS BIBLIOGRÁFICAS}

Anderson, T. (2005) Distance learning-Social software's killer ap? Consultado el 17 de junio de 2009. http://auspace.athabascau.ca:8080/dspace/bitstream/2149/2328/1/distance_learning.pdf.

Arsun, O. I. y Kuru, S. (eds.) (2006) iCamp. Innovative, inclusive, interactive E intercultural learning campus. Draft Exploitation Model. Consultado el 12 de julio de 2009. http://www.icamp.eu/wp-content/uploads/2007/05/d52_icamp_exploitation-modeldraft.pdf.

Bajtelsmit, J. W. (1990) Study methods in distance education: a summary of five research studies, en Moore, M. G. (ed.) Contemporary Issues in American Distance Education. New York, Pergamon Press, 181-191. 
Barkley, E. F.; Cross, K. P. y Major, C. H. (2005) Collaborative learning techniques: A handbook for college faculty. San Francisco, Jossey-Bass.

BarRy, M. y RunYAN, G. (1995) A review of distance learning studies in the U.S. Military. The American Journal of Distance Education, 9 (3), 37-47. (Texto electrónico en Biblioteca UNED).

Basu, S. (2005) If there is no significant difference, why should we care? The Journal of Educators Online, 2 (2). Consultado el 23 de junio de 2009. http://www.thejeo.com/ Basu\%20Conger\%20Final.pdf.

Bates, A. W. y Poole, G. (2003) Effective teaching with technology in higher education Foundations for success. San Francisco, Jossey-Bass.

Bernard, R. M.; Abrami, P. C.; Lou, Y.; Borokhovski, E.; Wade, A; Wozney, L.; Andrew, P.; Fiset, M. y Huang, B. (2004) How does distance education compare with classroom instruction? A meta-analysis of the empirical literature. Review of Educational Research, 74 (3), 379-439. (Texto electrónico en Biblioteca UNED).

Boyd, D. (2007) The significance of social software, en Burg, T. N. y Schmidt, J. (eds.) BlogTalks reloaded: Social software research \& cases. Norderstedt, Books on Demand, 15-30. Consultado el 21 de junio de 2009. http://www.danah.org/papers/BlogTalksReloaded.pdf.

Bragg, A. B. (2007) Reflections on pedagogy: Reframing Practice to Foster Informal Learning With Social Software. Consultado el 20 de marzo de 2009. http://matchsz.inf.elte.hu/tt/ docs/Anne20Bartlett-Bragg.pdf.

BRown, G. y WACK, M. (1999) The difference frenzy and matching buckshot with buckshot. The Technology Source. Consultado el 12 de mayo de 2009. http://technologysource. org/?view=article\&id=320.

Chambers, J. A. (2002) This time, we get to do it right. Community College Week, 14 (20), 8-9. (Texto electrónico en Biblioteca UNED).

Chang, T. M. (1983) Distance Learning: On the Design of an Open University. Boston, KluwerNijhoff-Publishing.

Chester, A. y Gwynne, G. (1998) Online Teaching: Encouraging Collaboration through Anonymity. Journal of Computer Mediated Communication, 4 (2). Consultado el 23 de junio de 2009. http://jcmc.indiana.edu/vol4/issue2/chester.html.

Clark, R. (1983) Reconsidering research on learning from media. Review of Educational Research, 53 (4), 445-460.

- (1994) Media will never influence learning. Educational Technology Research and Development, 42 (2), 21-29.

Daniel, J. S. (1996) Mega-universities and Knowledge Media: Technology Strategies for Higher Education. London, Kogan Page.

Davies, T. G. y Quick, D. (2001) Reducing distance through distance learning: The community college leadership doctoral program at Colorado State University. Community College Journal of Research E Practice, 25 (8), 607-620. Consultado el 13 julio, 2009. http:// www.informaworld.com/smpp/ftinterface $\sim$ content $=$ a $713837753 \sim$ fulltext $=713240930$.

Davis, I. (2005) Talis, Web 2.0 and All That. Internet Alchemy. Consultado el 27 de junio de 2009. http://internetalchemy.org/2005/07/talis-web-20-and-all-that.

Franklin, T. y Harmelen, M. (2007) Web 2.0 for Content for Learning and Teaching in Higher Education. JISC. Consultado el 23 de agosto de 2009. http://staff.blog.ui.ac.id/harrybs/ files/2008/10/web-2-for-content-for-learning-and-teaching-in-higher-education.pdf. 
García Aretio, L. (1986) Educación superior a distancia. Análisis de su eficacia. Badajoz, UNED-Mérida.

- (1987) Rendimiento académico y abandono en la educación superior a distancia. Madrid, ICE-UNED.

- (1994) Educación a distancia hoy. Madrid, UNED.

- (coord.) (1997) Investigar para mejorar la calidad de la Universidad. Madrid, UNED.

- (1999) Historia de la educación a distancia. Revista Iberoamericana de Educación a Distancia (RIED), 2 (1), 11-40. Consultado el 17 de mayo de 2009. http://www.utpl. edu.ec/ried/images/pdfs/vol2-1/historia_de_la_eduacion.pdf.

- (2001) Educación a distancia. De la teoría a la práctica. Barcelona, Ariel.

- (2009) ¿Por qué va ganando la educación a distancia? Madrid, UNED.

Hiltz, S. R.; Zhang, Y. Z. y Turoff, M. (2002) Studies of Effectiveness of Learning Networks, en Mayadas, F.; Bourne, J. y Moore, J. C. (eds.) Elements of quality online education, vol. 3 in the Sloan-C Series. Needham, MA, Sloan-C.

KaYE, A. (1981) Origins and structures. Distance Teaching for Higher and Adult Education. Milton Keynes, Open University Press.

KeEgan, D. (1996) Foundations of distance education. New York, Routledge.

Klamma, R. y otros (2007) Social software for life-long learning. Journal of Educational Technology and Society, 10 (3), 72-83. Consultado el 23 de junio de 2009. http://www.ifets. info/journals/10_3/6.pdf.

Krajnc, A. (1988) Social isolation and learning effectiveness in distance education. Ziff Papiere 71. Hagen, FernUniversitat. Consultado el 12 de agosto de 2009. http://www.eric. ed.gov/ERICDocs/data/ericdocs2sql/content_storage_01/0000019b/80/1e/2e/46.pdf.

Levin, A. (2004) Social software: What's new. Consultado el 17 de febrero de 2009. http:// www.corante.com/many/archives/2004/10/18/social_software_whats_new.php.

LÉvy, P. (2004) La inteligencia colectiva. Washington, Organización Panamericana de la Salud. Consultado 1 de julio de 2009. http://inteligenciacolectiva.bvsalud.org/public/documents/pdf/es/inteligenciaColectiva.pdf.

Lockee, B.; Moore, M. y Burton, J. (2001) Old Concerns with New Distance Education Research. EDUCAUSE Quarterly, 24 (2), 60-62. Consultado el 23 de junio de 2009. http:// www.coe.ufl.edu/Courses/eme5054/Foundations/Articles/Burton.pdf.

Matthews, D. (1999) The origins of distance education and its use in the United States. T.H.E. Journal, 27. Consultado el 17 de julio de 2009. http://www.questia.com/googleScholar. qst?docId $=5001313351$.

Means, B.; Toyama, Y.; Murphy, R.; Bakia, M. y Jones, K. (2009) Evaluation of Evidence-Based Practices in Online Learning: A Meta-Analysis and Review of Online Learning Studies. Washington, U.S. Department of Education. Consultado el 17 de agosto de 2009. http:// www2.ed.gov/rschstat/eval/tech/evidence-based-practices/finalreport.pdf.

Moore, M. y Thompson, M. M. (1997) The effects of distance learning. Pennsylvania, American Center for the Study of Distance Education, Pennsylvania State University.

O'Really, T. (2005) What Is Web 2.O. Design Patterns and Business Models for the Next Generation of Software. Consultado el 30 de agosto de 2009. http://oreilly.com/web2/ archive/what-is-web-20.html.

PAuL, R. (1990) Towards a new measure of success: developing independent learners. Open Learning, 5 (1), 31-38. Ed. en español: Hacia una autonomía del alumno: nueva pauta para medir los logros de una institución de enseñanza abierta, en MarTín, E. y AHIJADO, M. La educación a distancia en tiempos de cambio. Madrid, De la Torre, 43-60. 
PhIPPS, R. y MERISOTIS, J. (1999) What's the difference? A review of contemporary research on the effectiveness of distance learning in higher education. Washington DC, The Institute for Higher Education Policy. Consultado el 7 de agosto de 2009. http://www.ihep.org/ assets/files/publications/s-z/WhatDifference.pdf.

Resnick, D. (2000) The Virtual University and college life: Some unintended consequences for democratic citizenship. First Monday, 5 (8). Consultado el 17 de junio de 2009. http://131.193.153.231/www/issues/issue5_8/resnick/index.html.

RichARDSON, W. (2006) Blogs, wikis, podcasts, and others powerful webtools for classrooms. Thousand Oaks, CA, Corwin Press.

Rivard, N. (2002) Yes-online learning works. University Business, 5 (6), 20-21. (Texto electrónico en Biblioteca UNED).

Rourke, L.; Anderson, T.; Garrison, D. R. y Archer, W. (2001) Assessing social presence in asynchronous, text-based computer conferencing. Journal of Distance Education. Consultado el 14 de agosto de 2009. http://auspace.athabascau.ca:8080/dspace/bitstream/2149/732/1/Assessing\%20Social\%20Presence\%20In\%20Asynchronous\%20Textbased\%20Computer\%20Conferencing.pdf.

Russell, T. L. (2001) The No Significant Difference Phenomenon: A Comparative Research Annotated Bibliography on Technology for Distance Education. Montgomery, AL, IDECC.

SABA, F. (2000) Research in distance education: a status report. International Review of research in Open and Distance Learning, 1 (1), 2-9. Consultado el 27 de junio de 2009. http://www.irrodl.org/index.php/irrodl/article/view/4/24.

Schramm, W. (1962) What We Know About Learning from Instructional Television, en Educational television: The next ten years. Stanford CA, The Institute for Communication Research, Stanford University. Consultado el 17 de mayo de 2009. http://www.questia. $\mathrm{com} /$ PM.qst?a $=$ o\&d $=58715345$.

Shachar, M. y Neumann, Y. (2003) Differences between traditional and distance education academic performances: A meta-analytic approach. Consultado el 17 de junio de 2009. http://www.scribd.com/doc/3569335/Differences-Between-Traditional-and-DistanceEducation-Academic-Performances.

Siemens, G. (2004) Conectivismo: una teoría del aprendizaje para la era digital. Consultado el 27 de junio de 2009. http://www.diegoleal.org/docs/2007/Siemens(2004)-Conectivismo.doc.

Sigales, C. (2002) El potencial interactivo de los entornos virtuales de enseñanza y aprendizaje en la educación a distancia. Consultado el 18 de agosto de 2009. http://www.uoc. edu/web/esp/art/uoc/sigales0102/sigales0102.html.

Styles, C. (2006) How Web 2.0 will change history. Consultado el 17 de marzo de 2009. http://catherinestyles.files.wordpress.com/2006/08/web20history1.pdf.

TAIT, A. (2000) Students and attachment: the nature of electronic relationships. Adult Learning, 11 (10), 20-22. (Texto electrónico en Biblioteca UNED).

Weiler, K. (1991) Freire and a feminist pedagogy of difference. Harvard Educational Review, 61 (4), 449-474. (Texto electrónico en Biblioteca UNED). 